\title{
Embryonal Rhabdomyosarcoma of Conjunctival Tissue and Musculus Rectus Medialis in a 14 Years-0ld Girl
}

\author{
Stefan Bittmann*, Elisabeth Luchter, Elena Moschüring-Alieva, Gloria Villalon \\ Department of Pediatrics, Ped Mind Institute (PMI), Germany
}

*Corresponding author: Stefan Bittmann, Department of Pediatrics, Ped Mind Institute (PMI), Germany.

To Cite This Article: Stefan Bittmann. Embryonal Rhabdomyosarcoma of Conjunctival Tissue and Musculus Rectus Medialis in a 14 Years-Old Girl. Am J Biomed Sci \& Res. 2019 - 5(3). AJBSR.MS.ID.000909. DOI: 10.34297/AJBSR.2019.05.000909.

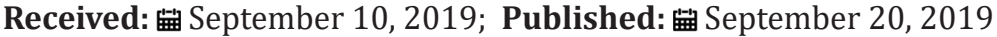

\begin{abstract}
Rhabdomyosarcoma is the most common soft tissue sarcoma in childhood and adolescence and is a tumor of the skeletal muscles. We present a 14 years old girl with an embryonal rhabdomyosarcoma (pT1a, N0, M0) of conjunctival tissue treated with systemic polychemotherapy (CWS guidance, SOTISAR register, subgroup C). First complete remission was present in august 2019.
\end{abstract}

Keywords: Rhabdomyosarcoma; Child; Polychemotherapy; Tumor

\section{Introduction}

Soft tissue tumors are classified according to the tissue they contain: Muscles, fat, fibrous tissue, vessels and nerves. Rhabdomyosarcoma is the most common soft tissue sarcoma in childhood and adolescence and is a tumor of the skeletal muscles. It occurs at any anatomical site, but usually in the head, neck or urogenital tract, where there is usually relatively little skeletal muscle involved. The skeletal muscle is only involved in the extremities of the body. Rhabdomyosarcoma is malignant, while the benign variant, rhabdomyoma, is very rare. Rhabdomyosarcoma tends to appear as an expanding mass, leading to pain and symptoms associated with compression of adjacent structures. Metastases can lead to bone pain, lung nodule or pleural effusion breathing difficulties, anemia, thrombocytopenia and neutropenia with a 5-year survival rate of less than $30 \%$. In contrast, the disease treated with combined surgery, radiotherapy and chemotherapy has a 5 -year survival rate of over $80 \%$. The incidence of rhabdomyosarcoma in the US is 6 cases per 100,000 per year, and about $87 \%$ of patients are under 15 years of age. It is valuable for medical students to understand an example of a clinical case of pediatric embryonic rhabdomyosarcoma of conjunctival tissue.

\section{Case Report}

A 14 years old girl presented with an embryonal rhabdomyosarcoma of the left conjunctiva histologically diagnosed by tumor tissue biopsy in October 2018. Our case shows manifestation of tumor involvement in musculus rectus medalist of the left eye and the superior conjunctiva. Initial oncological checkup revealed no metastatic disease. ultrasonography revealed no lymph nodes cervically. MRT showed tumor involvement of the conjunctiva in nasal region and the musculus rectus medialis. Neurocranial involvement was not found. $\mathrm{X}$ ray of the lungs, electrocardiography and echocardiography were without any pathological signs. Systemic chemotherapy was performed with 9 blocks due to CWS guidance, Sotisar register and subgroup C. 9 blocks of vincristine, ifosfamide and actinomycin D were following till 4/2019. Complications were found in form of transient paresthesia (1./2. Block) and ptosis due to vincristine therapy after 3. /4. Block. First complete remission was found in $7 / 2019$. Foot dorsiflexion due to vincristine medication was found. Follicle stimulating hormone was high and controls were necessary. To date of the publication, the patient is well after the 9-block chemotherapy.

\section{Discussion}

Rhabdomyosarcoma in childhood is a malignant soft tissue tumor of mesenchymal origin. It accounts for about $3.5 \%$ of cancer cases in children aged 0 to 14 years and $2 \%$ in adolescents and young adults aged 15 to 19 years. The incidence is 4.5 cases per 1 million children, corresponding to about 350 cases per year, and $50 \%$ of cases are observed in the first decade of life. Men have a higher incidence of embryonic tumors and blacks have a slightly higher incidence of alveolar tumors. The frequency may depend on the histological subtype of rhabdomyosarcoma as follows: Embryonic: Patients with embryonic rhabdomyosarcoma are predominantly male (male to female ratio, 1.5). The peak frequency is in the age group from 0 to 4 years, with about 4 cases per 1 million children, with a lower rate in adolescents, about 1.5 
cases per 1 million adolescents. This subtype accounts for $57 \%$ of patients in the Surveillance, Epidemiology, and End Results (SEER) database. The incidence of alveolar rhabdomyosarcoma does not vary according to sex and is constant from 0 to 19 years of age, with about one case per 1 million children and adolescents. This subtype accounts for $23 \%$ of patients in the SEER database. Pleomorphic/ anaplastic, mixed and spindle cell subtypes each account for less than $2 \%$ of children with rhabdomyosarcoma. Most cases of rhabdomyosarcoma occur sporadically without the recognition of a predisposing risk factor, with the exception of the following.

A genetic association with Li-Fraumeni cancer susceptibility syndrome, DICER1 syndrome, Neurofibromatosis Type I, Costello syndrome (with germline mutations HRAS), Beckwith-Wiedemann syndrome (more frequently associated with Wilms' tumor and hepatoblastoma), Noonan syndrome, high birth weight and large size for gestational age are associated with an increased incidence of embryonic rhabdomyosarcoma was found. Prognostic factors in rhabdomyosarcoma are usually curable in most children with localized disease who receive combined modality therapy, with more than $70 \%$ of patients surviving 5 years after diagnosis. Recurrences are rare in patients who were alive and free of events after 5 years, with a 10-year late event rate of $9 \%$. However, relapses are more common in patients with inoperable disease at an inoperable site at diagnosis and in patients with metastatic disease at diagnosis. The prognosis for a child or adolescent with rhabdomyosarcoma is related to the following clinical and biological factors like age, children aged 1 to 9 years have the best prognosis, while younger and older people are less well off. In recent studies by the Intergroup Rhabdomyosarcoma Study Group (IRSG), the rate of 5-year undisturbed survival (FFS) was $57 \%$ for patients under one year of age, $81 \%$ for patients between 1 and 9 years of age, and $68 \%$ for patients over 10 years of age.

The five-year survival rates for these groups were $76 \%, 87 \%$ and $76 \%$, respectively. Historical data show that adults do less well than children (5-year overall survival [OS], 27\% $\pm 1.4 \%$ and $61 \%$ $\pm 1.4 \%$, respectively; $\mathrm{P}<.0001$ ). Embryonal rhabdomyosarcoma can consist of small round cells (myoblast-like) and spindle cells (myotube-like). Band-like or club-like cells can exist. The cytoplasm is fibrillar and eosinophilic, and cytoplasmic stripes may be visible; phosphatic acid (PTAH) staining can be used to help with demanding Z-band and sarcomere arrangements, but they are not observed in less well differentiated cases. Where no streaks are detected, immunohistochemistry is essential for diagnosis. Tumor cells may in some cases have an evacuated cytoplasm ("spider web cells"). Some multinuclear tumor cells may contain spherical eosinophilic intracytoplasmic inclusions. In the botryoid type of embryonic rhabdomyosarcoma (reported in the bladder and uterus) (see above) there is a similar composition of differentiated myoblasts mixed with poorly differentiated cells, and the stroma is often myxoid. Alveolar rhabdomyosarcomas consist of undifferentiated round cells arranged in leaves, mostly with alveolar formation due to multifocal loss of tumor cell cohesion.

Tumor cells tend to be smaller and more uniform than other subtypes. Pleomorphic tumors consist of highly pleomorphic tumor cells, including polynuclear, band-shaped cells in an abundant fibrous stroma. Nuclei contain prominent nucleoli, have marginalized chromatin, and are often arranged in parallel in polynuclear cells. There is moderate to pronounced anisocytosis and anisocaryosis with mitosis rates of $0-10$ per 10 high performance fields $(\times 400)$; a higher mitosis rate has been observed in more infiltrative neoplasms, but cellular pleomorphism has not been shown to be prognostic. Associated histologic changes may include inflammatory cell infiltrates (lymphoid cells and macrophages), bleeding, necrosis, and mineralization. Embryonal rhabdomyosarcoma of the conjunctiva is not often found and only few cases are published in literature [1-9]. Our case shows manifestation of tumor involvement in musculus rectus medalist of the left eye and the superior conjunctiva. Neurocranial involvement and manifestations were not found.

\section{References}

1. Pennington JD, Welch RJ, Lally SE, Shields JA, Eagle RC, et al. (2018) Botryoid Rhabdomyosarcoma of the Conjunctiva in a Young Boy. Middle East Afr J Ophthalmol 25(2): 111-114

2. Sarigül Sezenöz A, Karalezli A, Özkan Arat Y, Çoban G, Kiratli H, et al. (2017) Metastatic Embryonal Conjunctival Rhabdomyosarcoma in a 4-Year-Old Boy. Ophthalmic Plast Reconstr Surg 33(3S Suppl 1): S125-S127.

3. Mendez Mdel C, Muiños Y, Blanco G, Saornil MA, García-Alvarez C, et al. (2012) Embryonal rhabdomyosarcoma of the caruncle in a 4-year-old boy: case report. Arq Bras Oftalmol 75(3): 207-9.

4. Bhurgri Y, Mazhar A, Bhurgri H, Usman A, Malik J, et al. (2004) Orbital embryonal rhabdomyosarcoma in Karachi (1998-2002). J Pak Med Assoc 54(11): 561-565.

5. Brichard B, De Potter P, Godfraind C, Vermylen C (2003) Embryonal rhabdomyosarcoma presenting as conjunctival tumor. J Pediatr Hematol Oncol 25(8): 651-652.

6. Kodet R, Newton WA Jr, Hamoudi AB, Asmar L, Wharam MD, et al. (1997) Orbital rhabdomyosarcomas and related tumors in childhood: relationship of morphology to prognosis--an Intergroup Rhabdomyosarcoma study. Med Pediatr Oncol 29(1): 51-60.

7. Schworm HD, Boergen KP, Stefani FH. (1995) [The initial clinical manifestations of rhabdomyosarcoma]. Ophthalmologe. 92(3): 362-365.

8. Feng G, Yi Y, Zhang J, Li Y, Zheng H, et al. (1994) Clinicopathological analysis of rhabdomyosarcoma of the ocular adenexa. Yan Ke Xue Bao. 10(2): 125-128.

9. Eichholtz W (1974) Embryonic rhabdomyosarcomas in the form of epibulbar subconjunctival tumors (author's transl)]. Klin Monbl Augenheilkd. 164(3): 377-381. 